\title{
Calidad de vida en niños \\ y adolescentes con asma \\ en una población mexicana
}

\author{
Quality of life in children and teenagers \\ with asthma in a Mexican town
}

MCE Verónica Guajardo Balderas*, MCE Francisco Cadena Santos*, MCE Nohemi Selene Alarcón Luna*

MCE. Ma. De los Ángeles Alatorre Esquivel*, MCE Brenda Guadalupe Yáñez Castillo* y MES Alfonso Reyes Enríquez*.

*Maestro de Carrera tipo "G" de la Facultad de Enfermería de Nuevo Laredo, de la Universidad Autónoma de Tamaulipas

\section{Resumen}

Introducción: el propósito de este estudio fue evaluar la calidad de vida de niños y adolescentes con asma en la esfera biopsicosocial en una población mexicana.

Metodología: diseño del estudio descriptivo y correlacional. Muestreo no probabilístico, por conveniencia. Muestra de 108 participantes. Las mediciones fueron: Inventario de Calidad de Vida PedsQLTM y cedula de datos sociodemográficos.

Resultados: el 81.4\% de los participantes reportaron tres a más años de padecer asma, se identifico que 35.2\% habían estado internados de una a dos veces en el último año. Las subescalas de funcionamiento físico (33.27\%) y la emocional

Quality of life in children and teenagers with asthma in a Mexican town

Introduction: the purpose of this study was to evaluate the quality of life of children and teenagers with asthma on the biopsychosocial sphere in a Mexican town.

Methodology: design of descriptive study and co relational, not probabilistic sample for convenience. A sample with 108 participants. The measurements were: quality life inventory PedsQL and social demographic data schedule.

Results: $81.4 \%$ of participants reported 3 thru more years suffering asthma, a $35.2 \%$ was indentified were in hospitals once to twice during the last year. The sub-scales of physical
(33.24\%) son más altas que la social y la escolar. La consistencia interna del instrumento PedsQL ${ }^{\mathrm{TM}}$ es aceptable con un Alpha de Cronbach de 86

Conclusiones: la media de calidad de vida de niños y adolescentes es alta en las subescalas de funcionalidad física y emocional. El Instrumento de Calidad de Vida en Pediatría, reportó confiabilidad aceptable y puede utilizarse en población mexicana. Se recomienda considerar en estudios con población pediátrica sean realizados en diversos periodos del año para precisar mejor la variable.

Palabras Clave: Calidad de Vida, Asma. Niños, Adultos.

\section{Abstract}

functioning (33.27\%) and emotional (33.24\%) are higher than social and scholar. The internal consistency of the instrument PedsQL is acceptable with an alpha Cronbach of .86.

Conclusions: the average of quality of life of children and teenagers is high on the sub-scales of physical functionality and emotional. The quality of life in pediatrics instrument, reported acceptable trust and can be used on Mexican population. Is recommended to consider in studies with pediatric population to be made in different seasons of the year to precise the variable.

Key Words: Quality of Life, Asthma,Children, Teenagers 


\section{INTRODUCCIÓN}

El asma es la enfermedad crónica más frecuente en la edad pediátrica con claro incremento en su prevalencia en las últimas décadas'. El Sistema Nacional Epidemiológico publico que en México la incidencia de asma de 1996 fue de 239.7 mientras que para el 2003 fue de 298.1 por 100000 habitantes observándose un incremento de la incidencia del $19.6 \%$, al realizar una proyección hasta 2007 se tuvo una tasa del 308.2 por 100000 habitantes con un incremento del $22.4 \%$. Por entidad federativa los estados que presentan las tasas más altas desde 1998 hasta el 2003 son Yucatán, Quintana Roo, Tabasco y Tamaulipas. En el 2004 hasta la semana 19 Tamaulipas obtuvo una tasa de 211.0 por 100000 habitantes $^{2}$.

Nuevo Laredo, Tamaulipas es una ciudad fronteriza cuyas principales fuentes de trabajo son las maquiladoras textiles, empresas que provocan emanación de polvos y sustancias que afectan el aparato respiratorio, constituye un potencial para que sus pobladores presenten enfermedades pulmonares.

Los polvos y sustancias alérgenos pueden desencadenar asma, como en el caso de Ciudad Juárez, Chihuahua, ciudad fronteriza con características similares a las de Nuevo Laredo, que presenta una prevalencia de asma del $20 \%{ }^{3}$.

El asma es una enfermedad bronquial crónica caracterizada por inflamación, imperreactividad, broncoconstricción y obstrucción con diversos grados de gravedad ${ }^{4}$.

Los tratamientos con frecuencia suelen ser molestos, por lo que los niños y adolescentes se sienten apenados de utilizar un inhalador frente a sus compañeros de clase o juego, así como de que se les vea con una crisis de tos y secreciones; este hecho aunado a la sintomatología, sin duda afecta la calidad de vida de los menores; por lo que deben aprender a vivir con su enfermedad de la mejor manera posible ${ }^{5}$.

La calidad de vida en pacientes con problemas crónicos es de interés particular, debido a que aunque en México se pronostique un aumento en la esperanza de vida, ésta debería acompañarse de condiciones biopsicosociales aceptables, que permitan a los niños y adolescentes realizar las actividades físicas, sociales y escolares diarias, así como gozar de bienestar emocional. Pljaskic-Karmenov, Djordjevic, Radic y Kamenov ${ }^{6}$ estudiaron la calidad de vida en 125 pacientes con asma sintomática, cuya edad estaba entre siete y 17 años. Respecto al género 40 eran mujeres y 85 hombres, el promedio de edad en general fue de 12.14 años $(\mathrm{DE}=3.11)$. Los pacientes reportaron que los síntomas del asma les hacían levantarse durante la noche, inclusive levantarse más temprano, estos aspectos se relacionan con el funcionamiento físico.

La Asociación Española de Neumología Pediátrica7 reporto un estudio que tenía como objetivo medir la calidad de vida en un grupo de pacientes pediátricos con asma y analizar qué subescalas de la calidad de vida estaban más afectadas. Se estudiaron a 57 participantes, se reportó que la media global de calidad de vida, en los varones fue de 5.99 y en las mujeres de 5.62; las actividades que los niños señalaron que el asma les había molestado más fueron: correr $65 \%$, jugar fútbol $47 \%$, jugar en el recreo $28 \%$ y andar en bicicleta $22 \%$. En cuanto a las actividades de la vida diaria $10 \%$ reportaron tener algunos problemas para caminar, $16.47 \%$ tener dolor o malestar, $8.23 \%$ estar algo ansioso o deprimido y estar muy ansioso o deprimido solamente $1.17 \%$. La media en la limitación de la actividad en la población general fue de 5.52, en la función emocional fue de 6.23 y en la subescala de síntomas de 5.72 (no se proporcionan desviaciones estándar).

La definición de calidad de vida, con frecuencia se asocia al concepto de salud de la Organización Mundial de la Salud que dice que "es el completo bienestar físico, mental y social y no sólo la ausencia de enfermedad", por tal razón algunos conceptos de calidad de vida tienen como subescalas el funcionamiento físico, social y emocional ${ }^{8}$.

En la experiencia de los autores han observado que niños y adolescentes con asma se ven más afectados en las subescalas funcional y emocional. En relación a lo físico los niños y adolescentes se ven limitados para llevar a cabo actividades relacionados a correr, jugar fútbol, ejercicio y a actividades básicas de la vida diaria. Por lo que el propósito de este estudio esta dirigido a Evaluar la calidad de vida de niños y adolescentes con asma en la esfera biosicosocial en una población mexicana.

\section{METODOLOGÍA}

El diseño del estudio fue descriptivo y correlacional ${ }^{9}$. La población de estudio la conformaron niños y adolescentes medicamente diagnosticados con asma y que asisten a instituciones educativas federales (primarias y secundarias). El muestreo fue no probabilístico, por conveniencia. El tamaño de muestra de 108 participantes; calculado para una diferencia de medias, con un universo de 163 pacientes, error de .05 y nivel de confianza de $95 \%$.

Las mediciones utilizadas fueron un instrumento y una cedula de datos sociodemográficos; el instrumento utiliza- 
do fue el Inventario de Calidad de Vida PedsQL ${ }^{\mathrm{TM} 10}$ es un instrumento con cuatro subescalas que son: a) funcionamiento físico, b) funcionamiento emocional, c) funcionamiento social y d) funcionamiento escolar. La consistencia interna del instrumento reportada por el autor oscila entre .78 a .92.

Previo al consentimiento informado se aplicó la cédula de datos sociodemográficos, a fin de contar con el perfil de los participantes. La cédula contiene edad en años cumplidos del niño y adolescente, grado escolar, sexo, años de padecer el asma y el numero de internamientos en el ultimo año.

Para la recolección de los datos se llevo a cabo en una aula de la institución educativa facilitando la confidencialidad y la privacidad para contestar. El estudio conto con la aprobación del comité de ética de la institución de donde dependen los autores del estudio. De tal manera que se asegure el cumplimiento del reglamento de la Ley General de Salud en Materia de Investigación ${ }^{11}$.

\section{TABLA 1}

Datos sociodemográficos de la población.

\begin{tabular}{|c|c|c|}
\hline Variables & f & $\%$ \\
\hline \multicolumn{3}{|l|}{ Edad } \\
\hline $6-11$ & 31 & 28.7 \\
\hline 12 & 27 & 25.0 \\
\hline 13 & 26 & 24.1 \\
\hline $14-16$ & 24 & 22.2 \\
\hline \multicolumn{3}{|l|}{ Género } \\
\hline Masculino & 56 & 51.9 \\
\hline Femenino & 52 & 48.1 \\
\hline \multicolumn{3}{|c|}{ Escolaridad } \\
\hline De primero a tercero de primaria & 10 & 9.3 \\
\hline De cuarto a sexto de primaria & 22 & 20.4 \\
\hline De primero a tercer año secundaria & 76 & 70.3 \\
\hline \multicolumn{3}{|c|}{ Tiempo de padecer asma } \\
\hline De uno a menos de tres años & 20 & 18.6 \\
\hline De 3 a más años & 88 & 81.4 \\
\hline \multicolumn{3}{|c|}{$\begin{array}{l}\text { Numero de internamiento por } \\
\text { asma o complicaciones en el último año }\end{array}$} \\
\hline Sin internamiento & 61 & 56.4 \\
\hline De 1 a 2 internamientos & 38 & 35.2 \\
\hline De 3 a 7 internamientos & 9 & 8.4 \\
\hline
\end{tabular}

Fuente: Céd. de Datos Sociodemográficos $n=108$

\section{TABLA 2}

Valores estadísticos de las Subescalas del funcionamiento de calidad de vida que mide el PedsQL ${ }^{\text {TM }}$

\begin{tabular}{|c|c|c|c|c|}
\hline \multirow{2}{*}{$\begin{array}{l}\text { Subescalas } \\
\text { y Escala Total }\end{array}$} & \multicolumn{2}{|c|}{ Valores } & \multirow[b]{2}{*}{ C } & \multirow[b]{2}{*}{$\mathrm{DE}$} \\
\hline & Mín & Máx & & \\
\hline Funcionamiento Físico & .00 & 81.25 & 33.27 & 17.79 \\
\hline $\begin{array}{l}\text { Funcionamiento } \\
\text { Emocional }\end{array}$ & .00 & 85.00 & 33.24 & 20.78 \\
\hline Funcionamiento Social & .00 & 85.00 & 20.46 & 16.55 \\
\hline $\begin{array}{l}\text { Funcionamiento } \\
\text { Escolar }\end{array}$ & .00 & 90.00 & 28.98 & 18.95 \\
\hline Total & 1.14 & 68.18 & 30.89 & 15.26 \\
\hline
\end{tabular}

Fuente: Inventario de Calidad de Vida en Pediatría. $n=108$

\section{RESULTADOS}

El estudio fue realizado en 108 pacientes, los cuales asisten a Instituciones Educativas Federales; en el cuadro 1 se muestra que el $81.4 \%$ de los participantes reportaron tener de tres a más anos de padecer asma y se identifico que el $35.2 \%$ habían estado internados de una a dos veces en el último año.

En el cuadro 2 se observa que efectivamente las subescalas de funcionamiento físico (33.27\%) y la emocional (33.24\%) son más altas que la social y la escolar, inclusive son mayores que en la escala total del instrumento. Lo que significa que el padecer asma tiene más impacto en la esfera biológica y psicológica de la calidad de vida de los niños y adolescentes que en el área social.

La consistencia interna del instrumento PedsQL ${ }^{\mathrm{TM}}$ es aceptable con un Alpha de Cronbach de .86.

\section{DISCUSIÓN}

La confiabilidad obtenida del PedsQL ${ }^{\mathrm{TM}}$ es congruente a lo reportado por Varni ${ }^{10}$, con lo que se asume que el instrumento puede seguirse aplicando en población pediátrica mexicana.

Respecto al perfil de la población, la mayoría de los participantes fueron de escuelas secundarias, es decir que estaban en la edad de la adolescencia. El género de mayor predominio fue el masculino, esto es semejante a lo repor- 
tado por Pljaskic-Karmenov, Djordjevic, Radic y Kamenov6; señalan que el asma se presenta con mayor frecuencia en hombres que en mujeres. Los autores reportaron que su muestra la integraron 85 hombres y 40 mujeres.

En las subescalas de funcionalidad física y emocional e reportaron las medias mas altas de calidad de vida (aunque fueron menores al $60 \%$ ), estos datos difieren a lo reportado por Reichenberg ${ }^{12}$, quien señala que el $74 \%$ de sus participantes, mencionaron tener limitaciones para correr. Así mismo en el estudio reportado por la Asociación Española de Neumología Pediatrica ${ }^{7}$ se observa que el $65 \%$ y $47 \%$ tuvieron dificultades para correr y jugar respectivamente, esta situación de manera particular es semejante a lo encontrado en el estudio, pues más del $50 \%$ de los participantes reportaron estar afectados en estos aspectos de su funcionalidad.

Los porcentajes más bajos de afectación en la calidad de vida de los pacientes.

\section{CONCLUSIONES}

La media de calidad de vida de los niños y adolescentes es más alta en las subescalas de funcionalidad física y emocional que en la social y escolar. El Instrumento de Calidad de Vida en Pediatría, conocido con las siglas en ingles como

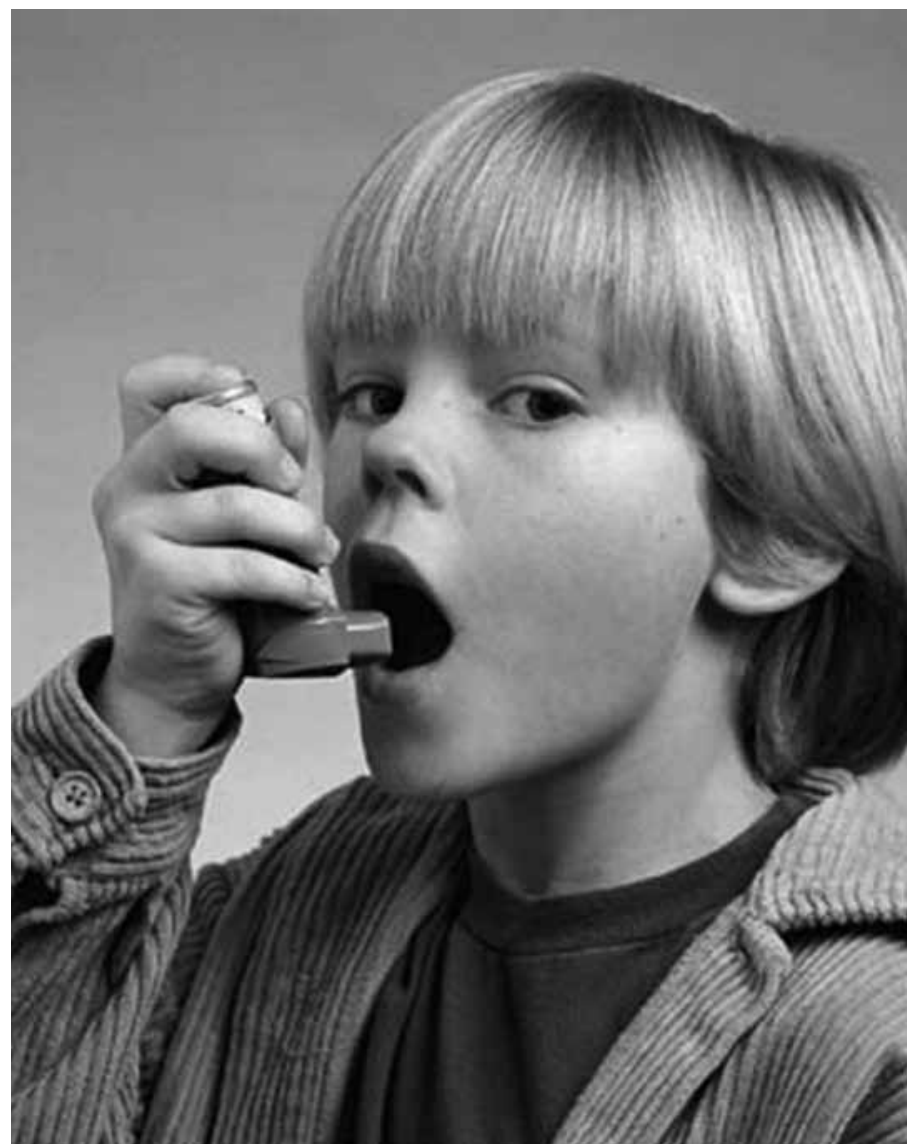

PedsQL ${ }^{T M}$ de Varni ${ }^{10}$, reportó confiabilidad aceptable y puede ser utilizado en población mexicana. El perfil de estudio respecto a edad y género, fue similar a lo reportado por otros autores, que consideraron niños y adolescentes en sus estudios. Se recomienda considerar dentro de otros estudios con población pediátrica, que estos sean realizados en diversos periodos del año para precisar mejor la variable.

\section{REFERENCIAS BIBLIOGRÁFICAS}

1. Lara-Pérez; E. A. Prevalecía del asma infantil al nivel del mar. Revista Alergia, Asma e Inmunológica Pediátricas. Vol. 10 No. 3 Mayo-Junio. México, 2001. pp. 88-92.

2. Sistema Nacional de Vigilancia Epidemiológica. Asma y estado asmático. Vol. 21. No. 30. Semana 30. 25 al 31 de Julio del 2004.

3. Barraza-Villarreal; A, y cols. Prevalencia de asma y otras enfermedades alérgicas en niños escolares de ciudad Juárez, Chihuahua. Salud Publica de México, 2001, 43(3), 433437.

4. Chapela-Mendoza; R. y cols. El asma, un problema de salud pública. Instituto Nacional de Salud Pública. Vol 3 No. 2 Febrero. México 2001.

5. Seipp C. Asthama attack: When "zero tolerance" collides with children's health 2002. Recuperado de htte: //www. reason .com

6. Pljaskic-Karmenov; S. S. y cols. Asthma Quality of Life as Marker of Disease Severity and Treatment Evaluation in School Children. Medicine and Biology, 2002, 9(2), 175-180.

7. Asociación Española de Neumología Pediatría. Valoración de calidad de vida en niños asmáticos. Recuperado el 19 de febrero de 2003 de http://www.Neumped.org/ calidad\%20de\%20vida.pdf.

8. Blasco S. A. Cuestionarios de calidad de vida: Aspectos teóricos. Alergología e Inmunológica Clínica. 2001, 16, 239-263.

9. Polit F. D, Hungler P. B. Investigación Científica en Ciencias de la Salud. (6 $6^{a}$ ed). Mc Graw-Hill Interamericana, México, 1987.

10. Varni J. W. Inventario de Calidad de Vida. PedsQL. Mapi research Institute, QoL Newsletter, 1998.

11. Leyes y Códigos de México. Ley General de Salud. Reglamento de la ley general de salud en materia de investigación para la salud. Capítulo I, III y V; Artículos 14, 17, 20, 21 y 58; Fracciones I, II, IV, VI, VII, VIII. 3ra. Ed. Porrúa. México, 1987.

\section{DIRECCIÓN PARA CORRESPONDENCIA}

Verónica Guajardo Balderas.: vguajardo@uat.edu.mx 